\title{
Large Scale Structure in Dark Matter and Galaxies
}

\author{
Nick Scoville \\ Astronomy Department, Caltech 105-24, Pasadena, CA 91125, USA
}

\begin{abstract}
Galaxy evolution and AGN growth in the early universe are believed to be strongly driven by merging (hierarchical growth) and galaxy dynamical interactions. Thus, a full exploration of the environmental influences is absolutely essential to understanding this early evolution. The Cosmic Evolution Survey (COSMOS, [Scoville et al. 2007a]) is specifically designed to probe the correlated coevolution of galaxies, star formation, active galactic nuclei (AGN) and dark matter (DM) largescale structures (LSS) over the redshift range $\mathrm{z}>0.5$ to 6 . The survey includes multi-wavelength imaging and spectroscopy from $\mathrm{X}$-ray to radio wavelengths covering a 2 square degree equatorial field. Photometric redshifts are derived using 34 photometric UV-IR bands for 800,000 galaxies with accuracy reaching $\sigma_{z} /(1+z)=0.7-1.6 \%$ for bright galaxies $\left(\mathrm{I}_{A B}=22-24 \mathrm{mag}\right)$.

Large scale structures have been traced in COSMOS from $\mathrm{z}=0.2$ to 2.5 in the baryons (from the galaxy density distribution) and in the dark matter to $\mathrm{z}=1.1$ (from weak lensing analysis of HST ACS images). These LSS extend over $20 \mathrm{Mpc}$ with total mass up to $\sim 10^{15} M_{\odot}$. The overall distribution of galaxy overdensities is similar with those predicted from the Millennium simulation. A trend for an increasing high overdensities at low $\mathrm{z}$ is clearly apparent in these data. At higher redshifts of $\mathrm{z} \sim 1$, there appears to be a significant discrepancy between the observations and the simulations - with the simulations exhibiting earlier development of high density structures than is seen in the observed galaxy distributions. The observed galaxy spectral energy distributions (SEDs) and star formation rates (SFRs) clearly depend systematically on both redshift and environmental density - early SED types and lower SFRs in denser regions and at lower redshift. This evolution is probably driven by the exhaustion of the ISM and by galaxy interactions, the latter being strongly correlated with regions of highest dark matter density. Strong evolution is seen the frequency of close pairs of galaxies - particularly for lower mass companions at projected separations $10-20$ kpe.
\end{abstract}

Keywords: cosmology, galaxy evolution

\section{INTRODUCTION}

Our understanding of the formation and evolution of galaxies and their large-scale structures (LSS) has advanced enormously over the last decade - a result of a phenomenal synergy between theoretical and observational efforts. Deep observational studies using the Hubble Space Telescope (HST) and the largest ground based telescopes have probed galaxy and AGN populations back to redshift $\mathrm{z}=6$ when the universe had aged less than 1 billion of its current 13 billion years. Just as remarkable is the enormous success of numerical simulations for $\Lambda$ CDM models in reproducing many of the current LSS characteristics, all starting from an initial, nearly uniform, hot universe! 
bright gal. $(1<22.5)$

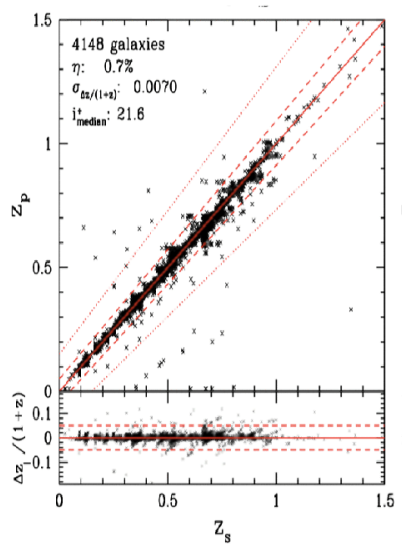

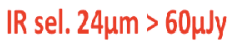

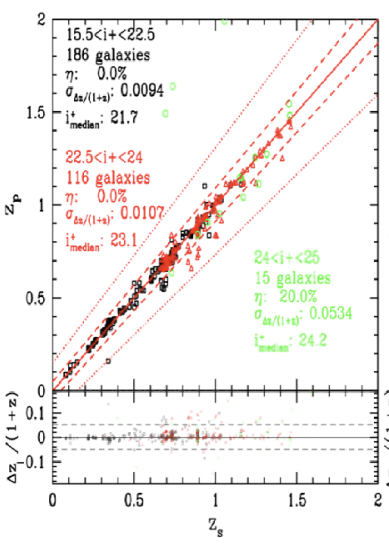

X-ray AGNs

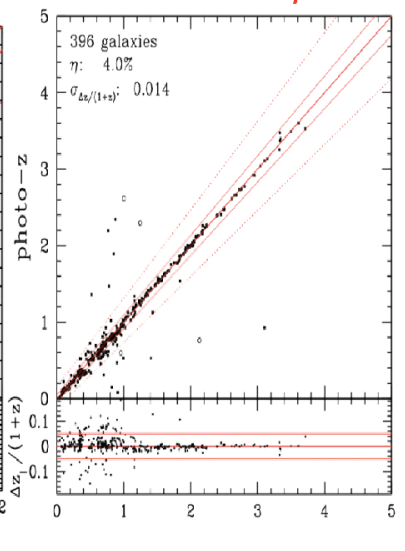

FIGURE 1. The present state-of-art photometric redshifts derived from the COSMOS 30 band photometry are shown for 3 samples of galaxies with confirming spectroscopic redshifts : bright galaxies with $i^{+}<22.5 ; 24 \mu \mathrm{m}$ selected galaxies [Ilbert et al. 2008] and X-ray selected AGN [Salvato et al.2008]. The accuracies are $\sigma_{z} /(1+z) \simeq 0.007,0.009$ and 0.014 respectively.

\section{THE COSMIC EVOLUTION SURVEY - COSMOS}

The COSMOS survey is the first survey encompassing a sufficiently large area that it can address the coupled evolution of LSS and galaxies, star formation and AGN. COSMOS is the largest HST survey ever undertaken - imaging an equatorial, 2 square degree field with single-orbit I-band exposures to a depth of $\mathrm{I}_{A B}=28 \mathrm{mag}(5 \sigma)$. Extensive multi- $\lambda$ ground and space-based observations of this field have been gathered spanning the entire spectrum from X-ray, UV, optical/IR, mid-infrared, $\mathrm{mm} / \mathrm{submm}$ and to radio with extremely high sensitivity imaging and spectroscopy (Figure 1). This full spectrum approach is required to probe the coupled evolution of young and old stellar populations, starbursts, the ISM (molecular and ionized components), AGN and dark matter. The multi- $\lambda$ approach is also required due to the differential redshifting of cosmic history and the prescence of dust obscuration in many of the most rapidly evolving galactic regions. The large area coverage of COSMOS is motivated to sample the largest structures existing in the local universe - smaller area coverage can lead to severe cosmic variance.

COSMOS detects $\sim 10^{6}$ galaxies and AGN sampling a volume in the high redshift universe ( $\mathrm{z}>0.5$ to 4$)$ approaching that sampled locally by the Sloan Digital Sky Survey (SDSS).

Determining the redshifts or lookback time of individual galaxies is clearly one of the most difficult and time consuming aspects of any cosmological evolution survey. Without high precision, large scale structures (LSS) become 'blurred' due to scattering of galaxies to different distances in the line of sight and for specific galaxies, their environment cannot be determined. COSMOS photometric redshifts are obtained from deep (mostly ground-based) imaging - Subaru, CFHT, UKIRT, and NOAO [Taniguchi et al. 2007, Capak et al. 2007a, Mobasher et al. 2007]. The photometric- 

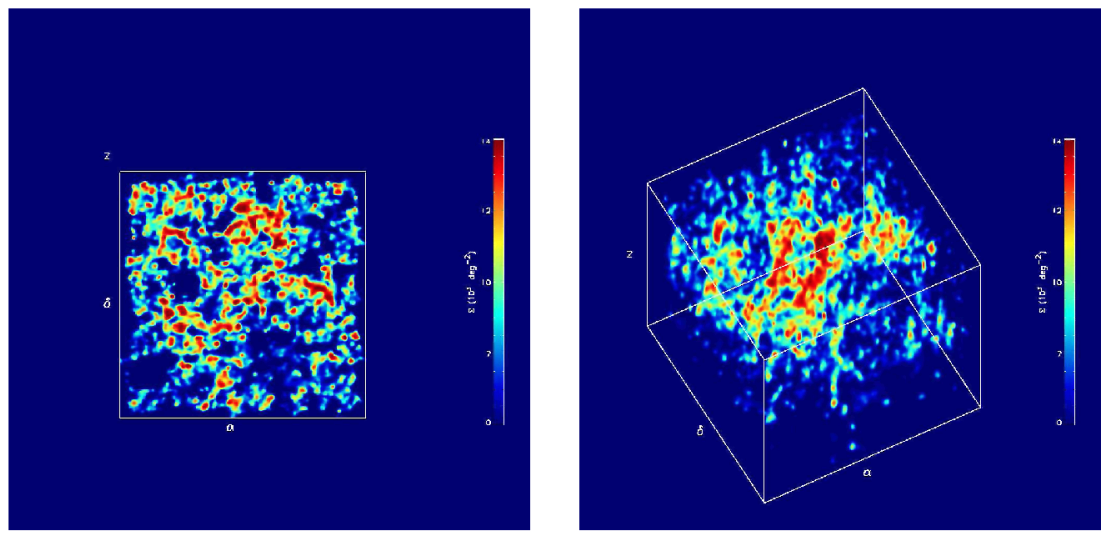

FIGURE 2. Left - Projected view on to sky of large scale structures (at $\mathrm{z}=0.2$ to 2.5 ) as derived from the overdensities of galaxies [Scoville et al. 2007b, Scoville et al. 2008] and Right-3-d view. The largest filaments can be tracked over $\sim 20 \mathrm{Mpc}$ in the the galaxies.

redshift accuracy is $\sigma_{z} /(1+z) \sim 1 \%$ for $4 \times 10^{5}$ galaxies [Mobasher et al. 2007, Ilbert et al. 2008]. These photometric redshifts are based typically on 34 bands of optical/IR data and the accuracy is unprecedented for photometric redshifts. Figure 1 shows the the derived photometric redshifts [Ilbert et al. 2008] plotted against the spectroscopic redshifts for three samples of galaxies (4330 galaxies with $\mathrm{I}_{A B}<22.5$ and 186 galaxies with $24 \mu \mathrm{m}$ MIPS detections). In both cases the, $\sigma_{z} /(1+z)<1 \%$ and there are very low catastrophic failure rates $(<1 \%)$.

\section{LARGE SCALE STRUCTURES - GALAXY OVERDENSITIES AND WEAK LENSING}

The environment or LSS in which a given galaxy resides is defined from the local number density of galaxies or from the DM density as determined from weak lensing. (The COSMOS HST imaging also provides measures of the close-in environment (from galaxy multiplicity and merger indicators such as tidal distortions) and larger-scale DM environment.)

A major goal of COSMOS has recently been realized - the first imaging of large scale structures in both the baryons from galaxy overdensities [Scoville et al. 2007b, Guzzo et al. 2007] and in the dark matter from weak lensing [Massey et al. 2007]. Figure 2 shows the structures seen in the galaxy overdensities (relative to the mean at each redshift) derived using adaptive smoothing on the galaxy densities in redshift slices [Scoville et al. 2007b]. The structures sizes range from 3 to $40 \mathrm{Mpc}$ and have masses up to $10^{15} M_{\odot}$. The left panel shows the distribution of structures projected on the sky for $\mathrm{z}=0.2$ to 2.5 and the right panel shows the full $3-\mathrm{d}$ distribution. In Figure 3 , the projected mass density derived from the weak lensing is shown in contour form [Massey et al. 2007] overlayed on the projected galaxy LSS (blue) and diffuse X-ray 


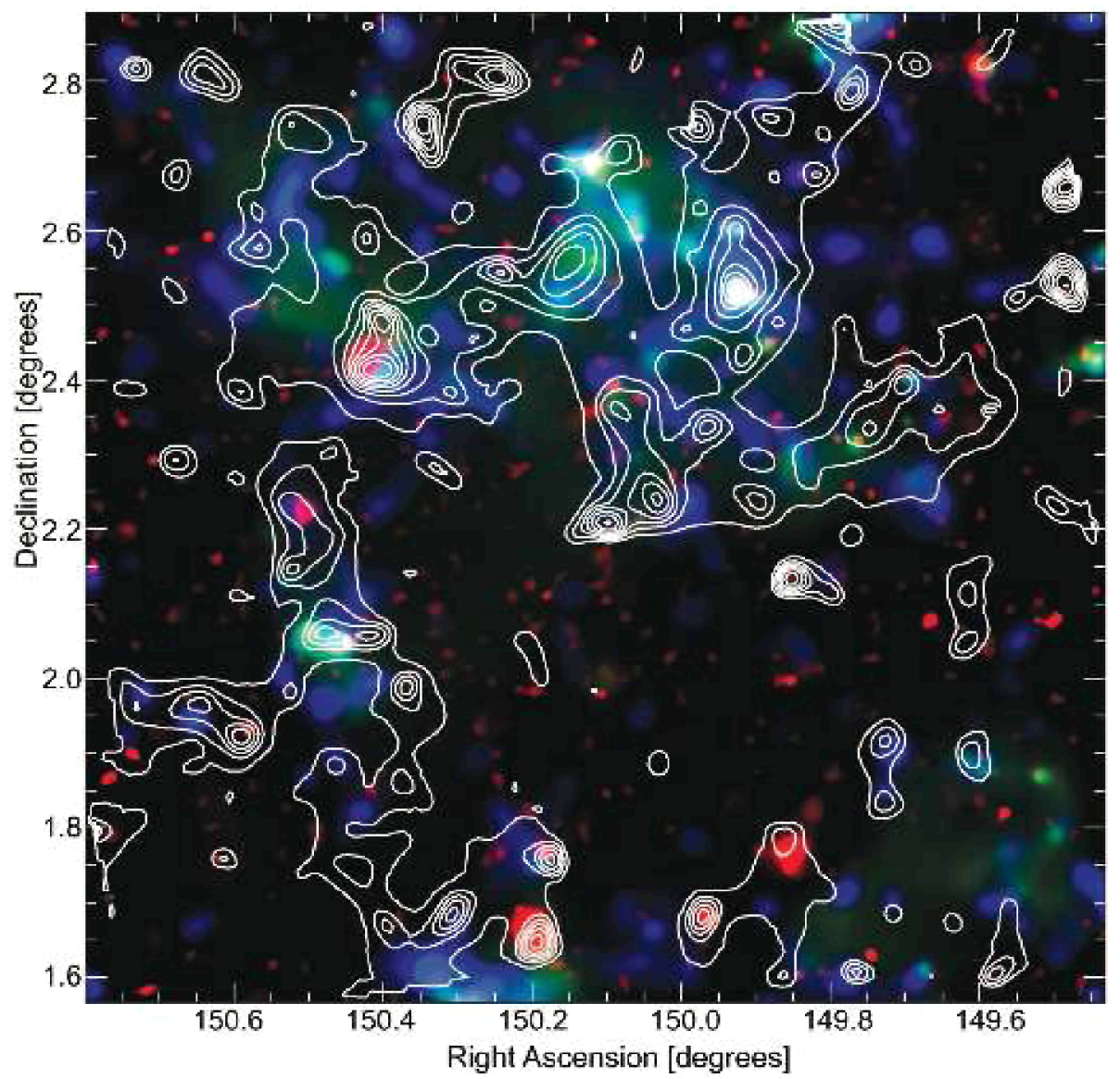

FIGURE 3. Comparison of the weak lensing dark matter mass map (contours) with galaxy LSS (blue/grayscale) and diffuse X-ray emission (red/grayscale) [Massey et al. 2007, Scoville et al. 2007b, Finoguenov et al. 2007]. This is the projected density distribution obtained from the full 3-d distribution derived using photometric redshifts for line of sight discrimination. Major structures shown here occur at $\mathrm{z}=0.25,0.5,0.73$ and 1.0 with sizes up to $\sim 40 \mathrm{Mpc}$ and total masses up to $10^{15} M_{\odot}$, similar to that of the COMA cluster.

emission (red) [Hasinger et al. 2007, Finoguenov et al. 2007]. In Figure 3 the redshift range is $\mathrm{z}=0.2$ to 1.2 . Overall there is excellent agreement between the LSS in the baryons and the dark matter; but there are a few notable discrepancies which deserve further verification and analysis.

A comparison between the observed LSS and those expected from simulations, specifically the Millennium simulation [Wang et al.2008], is shown in Figure 4. Here we plot the area filling fraction on the sky of LSS with different galaxy overdensities (relative to the mean density at each redshift). The fraction of material at higher overdensity is seen to increase systematically at lower redshift and the overall variation of the area filling 


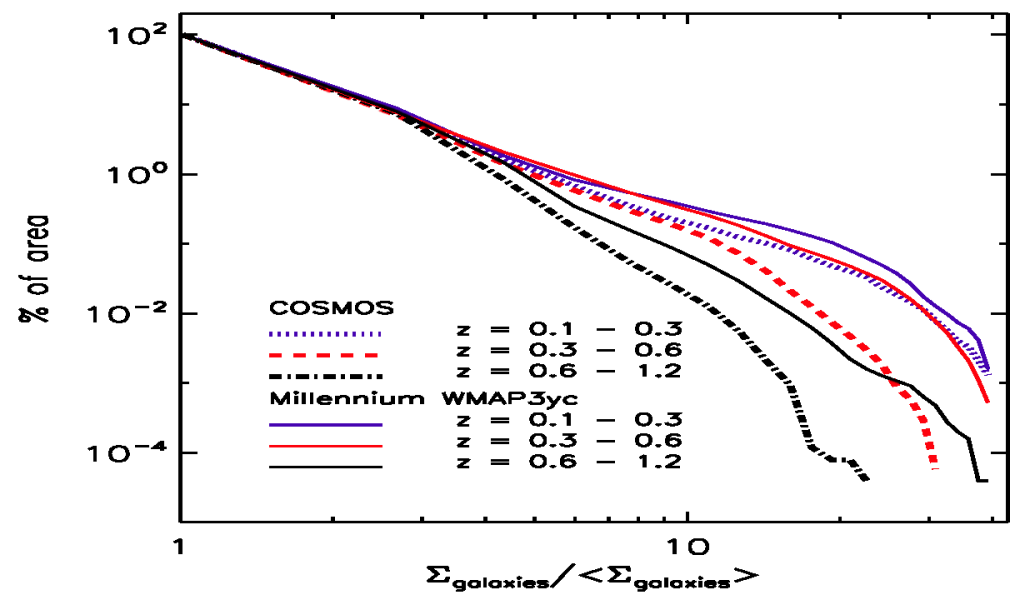

FIGURE 4. The fraction of the area of the sky as a function of overdensity ratio $\Sigma_{\text {galaxies }} /\left\langle\Sigma_{\text {galaxies }}\right\rangle$ relative to the mean at each redshift is shown for both the observed galaxies and from the Millennium COSMOS simulation (Wang et al.2008, processed with the same redshift uncertainties as the observed photo-z). A clear evolution to more high density LSS is seen at later epochs and the overall dependence of the area filling factor is similar to that in the simulation [Scoville et al. 2007b].

factor is remarkably similar to that predicted in the Millennium simulation.
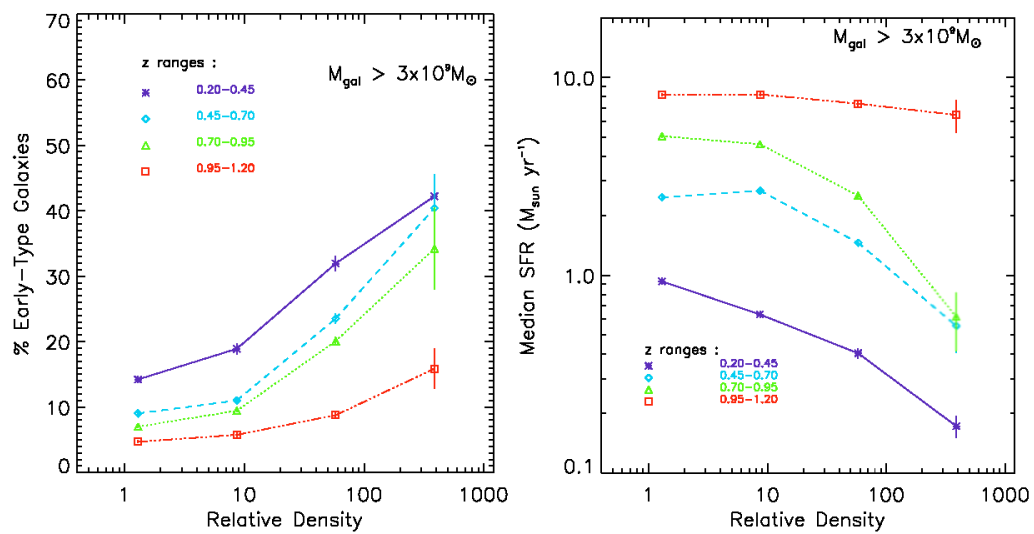

FIGURE 5. Left - The percentage of early type galaxies is shown as a function of environmental density for four redshift bins at $\mathrm{z}=0.2$ to 1.1 . The median star formation rate (SFR) derived from the UV SEDs is shown as a function of environmental density for four redshift bins at $\mathrm{z}=0.2$ to 1.1 [Scoville et al, 2007b]. The SFR clearly decreases to low $\mathrm{z}$ and at higher environmental density, very likely due to the exhaustion of the ISM and increasing galaxy interaction rates at higher density.

The dependence of morphology and galactic spectral energy distribution (SED) on environment has been followed in the $\mathrm{z}<1.1$ structures and into the field population [Scarlatta et al. 2007, Capak et al. 2007b, Scoville et al. 2007b]. Figure 5 shows the percentage of galaxies with early type SEDs as a function of redshift and local environmen- 
tal density (normalized relative to the mean at each redshift) [Scoville et al. 2007b]. With very small dispersions as a result of the large sample sizes and highly accurate SEDs, one clearly see the increase frequency of early types at low redshift and in denser environments. Similar correlations are seen in the median SFRs of galaxies (derived from the UV continuum) with respect to both redshift and epoch [Scoville et al. 2007b]. Much of this strong evolution is very likely due to the evolution of the merger rate from $z=1$ to the present [Scoville et al. 2008].

\section{ACKNOWLEDGMENTS}

I gratefully acknowledge the contributions of the entire COSMOS colaboration consisting of more than 100 scientists. The HST COSMOS Treasury program was supported through NASA grant HST-GO-09822 and Spitzer PID 20070 \& 30143. The status of these observational programs is continuously updated on the COSMOS web-site :

\section{http://www.astro.caltech.edu/ cosmos/.}

The major COSMOS datasets become publicly available in staged releases (following calibration and validation) through the web site for IPAC/IRSA :

\section{http://irsa.ipac.caltech.edu/data/COSMOS/}

\section{REFERENCES}

Capak et al. 2007a. Capak, P. et al. 2007, ApJSuppl.,172, 99

Capak et al. 2007b. Capak, P. et al. 2007, ApJSuppl., 172, 284

Finoguenov et al. 2007. Finoguenov, A. et al. 2007 ApJS, 172, 182

Guzzo et al. 2007. Guzzo, L. et al. 2007, ApJSuppl., 172, 254

Hasinger et al. 2007. Hasinger, G, et al. 2007, ApJSuppl,, 172, 29

Ilbert et al. 2008. Ilbert, O. et al.2008, Astro-ph 0809.2101I

Massey et al. 2007. Massey, R. et al. 2007, Nature,445, 286

Mobasher et al. 2007. Mobasher, B. et al. 2007, ApJSuppl., 172, 117

Salvato et al.2008. Salvato, M. et al.2008, Astro-ph 0809.2098S

Scarlatta et al. 2007. Scarlata, C. et al. 2007 ApJS, 172, 406

Scoville et al. 2007a. Scoville, N. et al. 2007a, ApJSuppl., 172, 1

Scoville et al. 2007b. Scoville, N. et al. 2007b, ApJSuppl., 172, 150

Scoville et al. 2008. Scoville, N. et al. 2008, ApJ, (in preparation)

Taniguchi et al. 2007. Taniguchi, Y. et al. 2007, ApJ Suppl., 172, 9

Wang et al.2008. Wang, J, et al.2008b, MNRAS, 384, 1301 